\title{
Instituições dos Estados, Educação dos Jovens e Analfabetismo: Um Estudo Econométrico em Painel de Dados
}

\author{
- JOILSON DIAS* \\ - Maria Helena ambrosio dias*
}

\begin{abstract}
RESUMO
O objetivo deste artigo é desenvolver um estudo empírico que considere as instituições dos Estados e seus impactos na educação dos jovens e na redução do analfabetismo. As instituições são entendidas como políticas educacionais, eficiência governamental (custo), estrutura da economia e mercado de alocação dos recursos humanos. A fundamentação teórica é baseada em Dias e McDermott (2006). Na estimativa empírica do modelo foram utilizados painéis de dados para os Estados brasileiros, período 199/-2000. Em geral, os resultados obtidos foram os seguintes: i) os investimentos em educação dos Estados são efetivos somente na redução de analfabetos e estão negativamente correlacionados com a educação dos jovens em nível de ensino médio; ii) os custos dos governos estaduais se apresentam como um fator negativo para a educação dos jovens que buscam escolaridade acima de oito anos; e, iii) o estímulo à acumulação de conhecimento de longo prazo nos Estados é heterogêneo, em função da taxa efetiva de retorno do capital humano e do estoque de capital humano com escolaridade acima de onze anos em cada Estado, ou seja, a estrutura do mercado de alocação de capital humano difere nos estados. Este último, juntamente com o grau de desenvolvimento da economia, nível tecnológico e de capital, demonstram ser os elementos preponderantes para a educação dos jovens e a redução dos níveis de analfabetismo no longo prazo.
\end{abstract}

\section{Palavras-Chave}

instituições, educação, analfabetismo, painel de dados, Estados

\begin{abstract}
The objective of this paper is to develop an empirical study that considers the aspects of the Brazilian States' institutions and their impact on youth education and on population illiteracy rate. We define institutions as being educational policies, government efficiency (cost), the structure of the economy and the market for human capital in each state. The theoretical model is borrowed from Dias and McDermott (2006). We test the model using a panel data approach for the years 199I-2000. In general our results are that: i) education investment made by each state showed to be significant and effective in curbing down the illiteracy rate; however, it showed to be negatively correlated in supporting youth high school education; ii) the States' government cost showed to have a negative influence on youth knowledge accumulation beyond eight years; iii) the rate of knowledge accumulation among states are heterogeneous because their differences in rate of return to education and the stock of human capital with eleven years or more of education. This last result together with the level of development, level of technology and physical capital, showed to be the foremost important element for youth knowledge accumulation and illiteracy rate reduction.
\end{abstract}

\section{KEYWORDS}

institutions, education, illiteracy, panel data, states

\section{JEL ClassificATION}

$015,011, \mathrm{C} 33$

\footnotetext{
+ Os autores agradecem o apoio recebido do CNPq e os comentários dos pareceristas anônimos.

* Professores Titulares do Departamento de Economia da Universidade Estadual de Maringá. Endereço para contato: Av. Colombo, 5790 - Maringá - PR. E-mails: jdias@uem.bremhadias@uem.br

(Recebido em agosto de 2006. Aceito para publicação em novembro de 2007).
} 


\section{$1 \quad$ INTRODUÇÃO}

Nas economias em geral, a acumulação de capital humano dos jovens é um fator preponderante na formulação das políticas educacionais. Esta importância aparece com destaque no artigo de Heckman (2000), que compara as políticas públicas de investimento na educação de jovens e de adultos com baixa qualificação. Como esperado, o resultado indica que o investimento na educação de jovens oferece um retorno mais elevado devido ao seu horizonte de recuperação dos recursos aplicados ser maior e porque a educação dos jovens é capaz de gerar novas habilidades neste capital humano. No entanto, ambos os programas de investimento são válidos, pois oferecem retornos à sociedade.

Com relação ao Brasil, os estudos de Gomes e Sobrinho (1992) e Barros e Mendonça (1998) podem ser destacados. Estes autores sugerem a necessidade de melhoria na qualidade, eficiência e equidade das políticas educacionais voltadas para os jovens. Os autores ainda enfatizam a importância de uma política ampla de acesso à educação aos jovens, como elemento fundamental para o desenvolvimento socioeconômico do Brasil. No entanto, quando os dados no período 1991-2000 são analisados, somente $22,26 \%$ dos jovens entre 15 e 17 anos obtiveram escolaridade acima de oito anos. ${ }^{1}$ Portanto, é de crucial importância compreender o papel exercido pelas instituições dos Estados no fomento à educação dos jovens, pois a educação dos jovens elimina a necessidade de políticas futuras de combate ao analfabetismo adulto, além de gerar ganhos de produtividade dos jovens relativamente a adultos analfabetos ao longo do tempo.

Neste artigo, as políticas e a eficiência do setor público compõem o que se denomina de instituições públicas. Mas, estas instituições não são as únicas responsáveis pela redução e/ou aumento da proporcionalidade de jovens educados e de adultos analfabetos. A estrutura da economia e seu mercado de trabalho são elementos importantes e formam neste artigo as instituições privadas. Por exemplo, Barros et alii (2001) encontrou que mercados mais atraentes a pessoas com menor qualificação tendem a induzir baixa acumulação da escolaridade média, ou seja, baixa acumulação de conhecimento. Neste caso, as diferenças entre as estruturas de mercados dos Estados podem fazer com que as políticas educacionais tenham seus efeitos maximizados e/ ou minimizados na acumulação de conhecimento dos jovens. Assim, procura-se aqui combinar tais elementos, de forma que se possa identificar seus efeitos individuais e obter estimativas mais robustas e específicas.

1 Fonte: www.ipeadata.gov.br. 
O conjunto formado pelos dois tipos de instituições, pública e privada, caracteriza as instituições dos Estados no agregado, que são compostas, neste estudo, da política educacional, da eficiência do governo, da estrutura de mercado de trabalho e do nível de desenvolvimento da economia. Portanto, o objetivo principal é avaliar o papel que estas instituições exercem na determinação da acumulação de capital humano dos jovens e na redução do analfabetismo entre os adultos. Em síntese, ganhos de produtividade representam o retorno econômico e social dos investimentos públicos realizados. No entanto, as políticas de investimento público podem estar desconsiderando este retorno econômico e social, ao serem insuficientes, ou até mesmo desestimulantes, especialmente quando a atuação das instituições privadas não é suficiente para incentivar a acumulação de conhecimento dos jovens.

Assim, este artigo investiga o papel exercido pelas instituições na geração e acumulação de capital humano de tal forma que políticas de educação dos jovens possam ser aprimoradas. Este trabalho está dividido em sete seções, sendo a primeira a introdução e a última a conclusão. A Seção 2 apresenta uma revisão sobre o papel das instituições no desenvolvimento econômico e suas diferentes definições na literatura. A Seção 3 aborda de forma breve o modelo teórico sobre o qual está embasado este trabalho. A Seção 4 efetua uma análise preliminar dos dados. A Seção 5 apresenta os resultados econométricos sob as condições mais apropriadas indicadas pelos testes. A Seção 6 refaz as estimativas anteriores, sob técnica econométrica alternativa, visando dar maior robustez aos resultados.

\section{INSTITUIÇÕES: CONCEITOS E PARTICIPAÇÃO NO PROCESSO DE ACU- MULAÇÃO DE CONHECIMENTO}

De acordo com Ahsan (2001), as instituições possuem diferentes conceitos, que podem ser resumidos no que se denomina de capital social, capital este capaz de influenciar o processo de acumulação de conhecimento e/ou educação. ${ }^{2}$ Este capital social pode ser analisado sob diversas formas ou diferentes papéis na economia. Por exemplo, para Hall e Jones (1999), as diferenças entre as economias são devidas ao capital social, representado pelos governos, infraestrutura natural e cultural. Como estamos tratando dos Estados brasileiros, as diferenças culturais são amenizadas, restando assim, dois elementos a serem considerados: governo e infraestrutura natural. A participação do governo será considerada em detalhes, pois pode, segundo nossa interpretação, representar dois aspectos importantes, os investimentos em educação e a eficiência dos governos (custo de operação). Quanto à infraestrutura natural, esta faz parte dos chamados efeitos fixos dos Estados. Neste caso, as técnicas

2 Coleman (1998) faz uma excelente descrição deste papel. 
a serem empregadas nas estimativas econométricas estão encarregadas de distinguir tais efeitos.

Um enfoque mais histórico sobre instituições é defendido por Sokoloff e Engerman (2000). Para os autores, o capital social está intimamente ligado às condições iniciais das economias, em especial à equidade ou à desigualdade de seu capital humano inicial. Assim, uma desigualdade inicial ainda pode estar sendo refletida nas políticas posteriores, gerando vantagens para um grupo em relação aos demais (nãoequidade), fazendo com que as diferenças iniciais persistam no tempo. Esta visão é intrigante, pois preconiza que o conhecimento adquirido por um grupo de pessoas em desvantagem não é suficiente para reduzir o problema de iniquidade, ou ainda, que o conhecimento adquirido pelo grupo em vantagem é suficiente para manter as barreiras. Tais barreiras podem ser mais bem compreendidas com o auxílio do estudo de Beltrão (2003). Em seu estudo sobre a evolução do analfabetismo no Brasil, a autora lembra que o analfabetismo age como uma barreira cognitiva que impede o avanço social deste grupo. O analfabetismo é, então, um fator limitador de desenvolvimento de um grupo dentro da sociedade, pois este ajuda a persistir as desigualdades no tempo e, portanto, pode estar agindo em acordo com o preconizado por Sokoloff e Engerman (2000).

Analisando esta persistência histórica, é como se o retorno econômico e social advindo de uma política de investimento mais intensa na acumulação de conhecimento dos jovens fosse desconsiderado, ou até preterido, ao longo do tempo. Mas será que esta negligência ou falha ainda continua na forma das instituições atuais dos Estados?

Se as instituições atuais existentes nos Estados, de alguma forma, refletem este aspecto histórico, observado por Sokoloff e Engerman (2000), então se espera um resultado negativo quando se considera o nível de escolaridade média dos indivíduos, e o grau de desenvolvimento da economia teria efeito inverso na redução do analfabetismo. Além disso, espera-se um impacto positivo da concentração de renda nos Estados brasileiros sobre o processo de acumulação de conhecimento dos jovens e adultos. A não observância destas características nos resultados pode indicar que os aspectos históricos não são predominantes ou que estão sendo paulatinamente reduzidos em importância.

Uma visão, dissociada do aspecto histórico, que está ligada às condições de políticas atuais e de mercado, idéia compartilhada aqui, está apresentada em Dias e McDermott (2006).

De acordo com os autores, as instituições são visualizadas como representantes de grupos, no caso, classe de empreendedores, que desempenha um papel crucial na 
acumulação de capital humano, e de rent seekers, que são indivíduos improdutivos que sobrevivem de parte da arrecadação do governo. Dessa forma, a classe empreendedora é que determina, por meio da remuneração do capital humano, o incentivo à acumulação deste capital. Portanto, esta classe age como um determinante do ponto de equilíbrio da decisão de educar dos indivíduos na economia. Por outro lado, o mercado exerce um importante papel, que é o de determinar a taxa de retorno implícita dos investimentos em educação, realizados pelos Estados e/ou indivíduos. No entanto, de acordo com Dias e McDermott (2006), a dimensão da classe empreendedora é determinada por políticas governamentais e pela eficiência dos governos.

No teste empírico do modelo, os autores utilizaram dados de painéis dos Estados brasileiros para explicar a proporção de jovens com idades entre 15 e 17 anos que possuíam escolaridade acima de oito anos. O principal resultado foi que as instituições privadas representadas pela proporção de empreendedores nas economias dos Estados são cruciais para a acumulação de capital humano dos jovens.

Como o objetivo neste trabalho é estender o trabalho de Dias e McDermott (2006), em especial seus aspectos empíricos, sem, contudo, alterar o modelo teórico, far-se-á uma breve exposição do modelo teórico presente no artigo original. Tal exposição será simplificada, com destaque na exposição do mecanismo através do qual as instituições públicas e privadas atuam no processo de acumulação de capital humano, especialmente dos jovens.

Em resumo, apesar da preponderância da figura central de um governo na intermediação do processo de arrecadar e alocar os recursos destinados à educação, este não determina exclusivamente as condições de acumulação de capital humano na economia, segundo o ponto de vista deste artigo. Aspectos históricos que podem ou não estar presentes, o que é chamado de efeitos fixos, e as condições atuais dos mercados formam, em princípio, um conjunto de instituições que podem interferir nesse processo. Apresentar o papel que estas instituições desempenham na acumulação do capital humano dos jovens e sua forma de atuação é objetivo a ser atingido na próxima seção.

\section{MODELO TEÓRICO: O PAPEL DOS EMPREENDEDORES NA ACUMU- LAÇÃO DE CAPITAL HUMANO}

O modelo teórico aqui apresentado é uma versão simplificada de Dias e McDermott (2006). Segundo os autores, as instituições de políticas governamentais ${ }^{3}$ conduzem

3 Instituições de políticas compreendem o Executivo, o Legislativo e o Judiciário. 
o processo de determinação e criação de um mercado para a acumulação de capital humano, ou seja, a proporção de empreendedores na economia. Em especial, as políticas educacionais determinam acesso ao capital humano dos indivíduos. A combinação de ambas, de forma eficiente, age como um elemento de propulsão da acumulação de capital humano no longo prazo, especialmente dos jovens.

Segundo a abordagem de Dias e McDermott (2006), os indivíduos da sociedade podem acumular capital humano e migrar para o setor moderno, ou podem se tornar empreendedores, ou ainda rent seekers, pessoas improdutivas que dependem dos recursos arrecadados dos Estados. As pessoas que se tornam empreendedores, além dos custos de entrada e constituição da firma, devem pagar taxas aos Estados, que mantêm pessoas improdutivas (rent seekers). Nesse modelo, as políticas educacionais operam com o intuito de reduzir o custo de migração, portanto operam diretamente no estímulo à acumulação de capital humano das pessoas. Esta política também gera como consequência no futuro o efeito autoalimentador, de melhoria da eficiência das instituições de políticas governamentais. No primeiro caso, os custos de migração representam o custo de educar na economia. No segundo caso, a eficiência das políticas educacionais, por sua vez, determina o fluxo de pessoas que adquirem capital humano e migram para o setor moderno da economia. A eficiência das instituições de políticas também implica a redução dos custos de se tornar empreendedor e, por conseguinte, o de reduzir subsídios aos rent seekers.

O modelo é composto de três setores. Um de produção tradicional, que se utiliza somente de trabalhador não educado, outro de produção moderna, que é baseado em capital humano, e o de acumulação de conhecimento. A migração dos indivíduos do setor tradicional para o setor moderno ocorre toda vez que o valor presente dos salários neste último setor ultrapassa os custos de migração. Dois detalhes são importantes nesta decisão. O primeiro é a taxa de retorno do capital humano, usada no cômputo do valor presente dos ganhos de se adquirir capital humano. Esta taxa depende do número de empreendedores, um número de empreendedores maior reduz a taxa de desconto, aumentando o valor presente dos ganhos futuros, que equivale a aumentar os ganhos presentes do capital humano. O segundo é o conjunto de parâmetros que representam as políticas educacionais do governo, que estão implícitas nos custos desta migração. Políticas educacionais ineficientes tendem a aumentar o custo de migração, o que reduz o processo de acumulação de conhecimento. A solução deste modelo implica duas funções de acumulação. A primeira determina o nível do capital humano da economia, que é dado por:

$$
\dot{h}=[\delta(E)-r] h
$$


Conforme a equação (1), a acumulação de capital humano depende da taxa de retorno $\delta(E)$ superar a taxa de mercado $(r)$, sendo que $\delta(E)$ está positivamente relacionada com o número de empreendedores $(E)$. A segunda função de acumulação determina o fluxo de pessoas qualificadas que migram para o setor moderno.

$$
\frac{\dot{L m}}{L m}=f\left(\frac{\delta(E) w(z) h-r \phi h^{\gamma}}{w_{T}}\right)
$$

Como podemos ver, a taxa de retorno do capital humano $\delta(E)$ vezes os salários do capital humano, $w(z) h$, tem que superar o custo de migração, $r \phi h^{\gamma}$. Os parâmetros $\phi$ e $\gamma$ representam a eficiência das políticas educacionais. Quanto menor forem os parâmetros, maior será o fluxo de pessoas qualificadas sendo absorvido pelo mercado. Por último, o salário do setor tradicional $\left(w_{T}\right)$ reduz este fluxo, especialmente quando ocorre uma demanda por mão de obra não-qualificada.

A última equação de acumulação do modelo deriva da condição de equilíbrio necessária para se tornar empreendedor. O valor presente dos ganhos como empreendedor, descontados os impostos e uma taxa fixa de instalação da firma, deve superar os ganhos de subsídios proporcionados pelo governo para os rent seekers. Este equilíbrio gera uma condição ótima entre a proporção de empreendedores e rent seekers que depende do nível de taxação na economia. Quanto maior a taxação, maior a arrecadação do governo proporcionalmente à renda existente, e maior a proporção de rent seekers vis à vis empreendedores na economia. $\mathrm{O}$ resultado final está representado na função que estabelece o fluxo de empreendedores, qual seja:

$$
\dot{E}=\theta_{E}\left[E^{*}(h, L m)-E_{o}\right]
$$

$\mathrm{Na}$ equação $(3), E^{*}(h, L m)$ representa o nível ótimo de empreendedores, dadas as políticas atuais, enquanto $\left(E_{0}\right)$ representa a quantidade mínima de empreendedores para garantir a existência de um setor moderno. Note que a quantidade ótima de empreendedores depende em última instância da seguinte função:

$$
E^{*}=v\left(z^{*}, \varepsilon, \tau\right) N
$$

Na expressão (4), $z^{*}$ é o estoque de capital humano na economia, $\varepsilon$ é o custo de implantação da firma e $\tau$ é o nível de tributos imposto às firmas. A variável $N$ representa a soma de empreendedores (E) e rent seekers (R) na economia. Políticas que aumentem o custo de abrir empresas e o nível de impostos reduzem a proporção ótima de empreendedores e aumentam a proporção de rent seekers. Como são as 
instituições de políticas que determinam estes dois parâmetros, estas exercem influência sobre a acumulação de conhecimento por meio da determinação do tamanho do mercado - classe empreendedora - que, por sua vez, absorverá o capital humano acumulado através das políticas educacionais.

Simulações deste modelo realizadas pelos autores, para um horizonte de 50 anos, demonstraram que a eficiência das políticas educacionais e a redução de impostos geram estímulos à acumulação de capital humano. A primeira, por reduzir o custo de aquisição de capital humano, e a segunda, por gerar demandas e melhorar a taxa de retorno do capital humano. Se houver necessidade de optar por uma das políticas, então a melhor escolha é a de redução de impostos, que demonstrou ser mais eficiente em comparação a outras políticas, mesmo quando se introduz um subsídio à educação. Simulações que consideram que a acumulação de capital humano gera maior eficiência nas instituições de políticas e, por conseguinte, reduzem o nível de taxação, posteriormente demonstraram ter efeito gradual e lento. Portanto, esta condição endógena é menos eficiente do que uma exógena, de mudança na atuação das instituições, para que estas se tornem mais eficientes a partir de um corte de impostos.

Dias e McDermott (2006) também estimaram este modelo para painel de dados dos Estados brasileiros, para o período 1996-2000. O objetivo foi explicar a proporção de jovens entre 15 e 17 anos com escolaridade acima de oito anos. Assim, os indicadores utilizados foram: proporção de empreendedores em cada Estado, média da escolaridade das pessoas acima de 25 anos, coeficiente Gini, gastos per capita com educação e percentual de gasto dos Estados com administração. Os autores reportaram como resultado final o papel preponderante da variável empreendedores, média da escolaridade e coeficiente Gini, sem, no entanto, encontrar significância para as variáveis de política educacional e de eficiência governamental.

Neste contexto, as estimativas a seguir estendem o trabalho descrito para explicar a proporção de analfabetos, percentagem de jovens que frequentam o primeiro e segundo graus e, principalmente, as proporções entre analfabetos e jovens educados e de jovens entre primeiro e segundo graus. Sob nosso ponto de vista, essas proporções retratam melhor as funções de acumulações propostas no modelo. Essas variáveis dependentes serão então funções das variáveis que apresentamos na introdução, que representam as instituições dos Estados. A principal razão para considerarmos a estrutura do mercado ao invés da variável proporção de empreendedores em nossas estimativas é que essa captura um grupo de pessoas com um conhecimento específico e, portanto, pode estar correlacionada a medidas da variável capital humano. 


\section{ANÁLISE DAS VARIÁVEIS DO MODELO ECONOMÉTRICO}

O modelo econométrico a ser estimado em painel de dados, procura averiguar o impacto das instituições de políticas governamentais sobre diferentes variáveis dependentes, relativas ao período 1991-2000. As variáveis dependentes a serem consideradas encontram-se descritas a seguir:

i) Analfa - percentagem da população analfabeta;

ii) Pjovpg - percentagem de jovens com idades entre 7 e 14 anos que frequentam o ensino fundamental (primeiro grau);

iii) Pjovsec - percentagem de jovens com idades entre 15 e 17 anos que têm acima de oito anos de escolaridade;

iv) Dpanalfa - razão entre as variáveis Analfa e Pjovpg;

v) Dpjovsec - razão entre Pjovsec e Analfa;

vi) Dpjovs - taxa efetiva de sucesso de qualificação de jovens com oito anos de escolaridade em relação à quantidade de jovens matriculados no primeiro grau.

Dessa forma, este conjunto de variáveis capta, sob diferentes ângulos, o processo de acumulação de conhecimento dos jovens e em relação à qualificação dos adultos nos Estados brasileiros. Assim, a variável Analfa mede o sucesso em qualificar as pessoas com um nível mínimo de educação (alfabetização); a variável Pjovpg é um indicador de qualificação mínima dos jovens, os indivíduos que se encontram na faixa etária de 15 a 17 anos no período atual. Já a variável Pjovsec é um indicador do nível de qualificação dos jovens atuais que ingressarão no mercado de trabalho futuro. A variável Dpanalfa, por sua vez, é uma sinalizadora do processo de qualificação de longo prazo na economia, uma vez que, quanto maior for a qualificação, menor será a quantidade de pessoas analfabetas e maior a quantidade de jovens frequentando a escola. A variável Dpjovsec procura medir o sucesso da educação, ou seja, procura medir a proporção de jovens com escolaridade acima de oito anos sobre a taxa de analfabetos, razão esta que se espera ser crescente. A variável Dpjovs é uma medida de sucesso do fluxo de educação dos jovens, ou seja, esta mede a progressão dos jovens concluintes do ensino fundamental (primeiro grau) para o ensino médio (segundo grau) de forma efetiva.

As variáveis independentes a serem utilizadas são divididas em dois tipos: variáveis de controle e explicativas. As primeiras procuram captar as heterogeneidades exis- 
tentes entre os Estados, seus efeitos fixos (infraestrutura etc). As variáveis do tipo explicativas procuram mostrar as mudanças ocorridas na variável dependente. As variáveis de controle são:

vii) Educ25c11 - percentagem da população com idade acima de 25 anos que tinha mais de 11 anos de escolaridade em 1991;

viii) Txresc - taxa geométrica média de retorno do capital humano anual no período $1991-2000 .^{4}$

As variáveis explicativas são:

ix) Pibh - Produto Interno Bruto dividido pela população e seu indicador de capital humano médio, nível de educação agregada da população acima de 25 anos educada $(E d u c 25)$;

x) Gini - índice de Gini, medida de distorção da distribuição da renda na economia;

xi) Efgov - eficiência governamental, medida através do logaritmo da razão entre o custo administrativo ( $A d m p c)$ e a diferença entre o custo total do Estado (Custopc) e os gastos com educação $(E d u c i n v p c)$, sendo todas as variáveis em termos reais per capita.

A utilização da variável Pibh, que visa captar o nível de desenvolvimento tecnológico e de capital dos Estados, exige algumas explicações adicionais. Ao dividirmos a produção total pelo nível de educação agregada, a variável resultante equivale a uma medida indireta dos níveis existentes de tecnologia e capital por trabalhador. Mais precisamente, temos o seguinte: $Y=A F(K, H, L)$, na qual $Y$ é a produção total de cada Estado; $A$ o nível de tecnologia; $K$ o nível de estoque de capital; $H$ o nível do estoque de capital humano - medido em anos de escolaridade da população acima de 25 anos (Educ25) e $L$ o número de trabalhadores. A divisão de $Y$ por $H$ e $L$ faz com que o produto por trabalhador, considerado o nível de capital humano, seja o seguinte: $Y_{h}=A f\left(k_{h}\right)$. Assim, a variável $Y_{h}$ ou Pibh, procura medir as diferenças entre $A$ e $k_{h}$ existentes entre os Estados. A utilização do nível agregado de educação na divisão é justificada pela teoria de Lucas (1988), e a inclusão da variável $Y_{h}$ na regressão é devida à sugestão de Temple (1999) para minimizar os efeitos da omissão das variáveis $A$ e $K$.

4 Txresc $=\sqrt[10]{H_{1991, i}}-H_{2000, i}$, onde $\mathrm{H}_{1991, i}$ e $\mathrm{H}_{2000, i}$ representam os valores médios em 1991 e 2000 do capital humano do Estado $i$. Fonte dos dados: www.ipeadata.gov.br. 
A fonte de dados é o IPEA - Instituto de Pesquisa Econômica e Aplicada. Os dados referem-se aos 27 Estados brasileiros $(\mathrm{n}=27)$, no período de dez anos $(\mathrm{T}=10)$, portanto, 270 observações. A tabela a seguir apresenta as principais características dos dados:

TABELA I - CARACTERÍSTICAS DOS DADOS

\begin{tabular}{lcccc}
\hline Variável & Média & Desvio Padrão & Mínimo & Máximo \\
\hline Analfa & $18,06 \%$ & $10,06 \%$ & $5,06 \%$ & $43,71 \%$ \\
Pjovpg & $90,43 \%$ & $5,81 \%$ & $70,26 \%$ & $99,44 \%$ \\
Pjovsec & $22,26 \%$ & $10,93 \%$ & $3,04 \%$ & $58,28 \%$ \\
Admpc & $\mathrm{R} \$ 230,53$ & $\mathrm{R} \$ 191,39$ & $\mathrm{R} \$ 40,70$ & $\mathrm{R} \$ 702,21$ \\
Educ25 & 5,06 & 1,15 & 2,55 & 8,24 \\
Gini & 0,57 & 0,037 & 0,42 & 0,66 \\
Educ25c11 & $5,72 \%$ & $3,28 \%$ & $1,72 \%$ & $17,42 \%$ \\
Dpjovs & $24,15 \%$ & $10,8 \%$ & $3,91 \%$ & $59,48 \%$ \\
Dpjovsec & $24,00 \%$ & $10,00 \%$ & $3,00 \%$ & $59,00 \%$ \\
Dpanalfa & $21,00 \%$ & $12,00 \%$ & $5,00 \%$ & $61,00 \%$ \\
Custopc & $\mathrm{R} \$ 446,30$ & $\mathrm{R} \$ 365,45$ & $\mathrm{R} \$ 95,97$ & $\mathrm{R} \$ 1.429,80$ \\
Educinvpc & $\mathrm{R} \$ 152,87$ & $\mathrm{R} \$ 109,34$ & $\mathrm{R} \$ 41,16$ & $\mathrm{R} \$ 774,90$ \\
Txresc & $5,81 \%$ & $0,61 \%$ & $4,66 \%$ & $7,39 \%$ \\
Pibh & $\mathrm{R} \$ 883,33$ & $\mathrm{R} \$ 338,85$ & $\mathrm{R} \$ 354,14$ & $\mathrm{R} \$ 1.804,80$ \\
\hline
\end{tabular}

Fonte: www.ipeadata.gov.br.

A Tabela 1 é de fácil compreensão; assim, far-se-á somente uma breve descrição das variáveis Analfa e Pibh. A média geral da variável Analfa é 18,06\%, portanto esta é a percentagem média da população analfabeta de cada Estado. $\mathrm{O}$ seu desvio padrão é de $10,06 \%$. O menor valor entre todas as observações é de $5,07 \%$ e o maior valor observado é 43,71\%. A média da variável Pibh é R $\$ 883,33$, que representa o produto por trabalhador considerando o nível de capital humano, igual ao produto efetivo de cada Estado por ano. O desvio padrão é de R $\$ 338,85$, sendo o menor valor o equivalente a R \$ 354,14 e o maior valor $\mathrm{R} \$ 1.804,80$. O desvio padrão é obtido entre os painéis, enquanto os valores mínimos e máximos são obtidos considerando todos os painéis no tempo.

Vale salientar que as variáveis Custopc (custo total do Estado por pessoa ao ano), Admpc (custo administrativo total do Estado por pessoa) e o Pibh (produto por capital humano per capita por ano) aparecem na tabela em valores reais por pessoa/ ano. 


\section{ESPECIFICAÇÃO E ESTIMATIVAS DO MODELO ECONOMÉTRICO}

O modelo econométrico a ser estimado em painel de dados é dado pelo seguinte conjunto de equações:

$$
\begin{aligned}
& y_{i t}=\beta^{\prime} x_{i t}+\xi_{i}+u_{i t} \\
& u_{i t}=\rho_{i} u_{i t-1}+\varepsilon_{i t} \quad \mid \rho_{i \mid<1}
\end{aligned}
$$

Na qual $y_{i t}$ representa a variável dependente; $\beta$ é o vetor de parâmetros a serem estimados (vetor kxl); $\boldsymbol{x}_{i t}$ representa o vetor de variáveis independentes (vetor kxl); $\xi_{i}$ compõe as características individuais a serem especificadas; e $\varepsilon_{i t}$ é a medida de erro. A equação (6) somente é válida para o caso de existência de autocorrelação, ou seja, quando $\rho_{i} \neq 0$.

O conjunto de testes em que os componentes dos erros são $E\left(\xi_{i} \xi_{j}\right)=\left\{0\right.$ se $i \neq j$ e $\sigma_{\xi}^{2}$ se $i=j\}$, ou seja, de que a variância entre os painéis dos Estados é constante, e da verificação da existência da autocorrelação entre painéis, agregada ou não, $\left|\rho_{i}\right|=|\rho|=0$, foi proposto por Baltagi e $\mathrm{Li}$ (1995). Os resultados destes testes usando as diferentes variáveis dependentes estão apresentados na Tabela 2.

A Tabela 2 mostra que o teste LM - Multiplicador de Lagrange rejeita a hipótese nula $\left(H_{0}\right)$ de variância constante e da não-existência de autocorrelação entre os painéis no tempo. Portanto, neste caso, como se esperava a priori, a especificação sugerida é para aplicação de um modelo de efeitos fixos.

\section{TABELA 2 - TESTE DOS COMPONENTES INDIVIDUAIS E DA AUTOCORRELAÇÃO}

\begin{tabular}{llc}
\hline Variáveis & Resultado do Teste & Probabilidade de H0 \\
\hline Analfa & $\mathrm{LM}[\operatorname{Var}(\mathrm{u})=0, \rho=0)=356,70$ & $\mathrm{P}(0,00)$ \\
Pjovpg & $\mathrm{LM}[\operatorname{Var}(\mathrm{u})=0, \rho=0)=212,32$ & $\mathrm{P}(0,00)$ \\
Pjovsec & $\mathrm{LM}[\operatorname{Var}(\mathrm{u})=0, \rho=0)=126,81$ & $\mathrm{P}(0,00)$ \\
Dpanalfa & $\mathrm{LM}[\operatorname{Var}(\mathrm{u})=0, \rho=0)=112,97$ & $\mathrm{P}(0,00)$ \\
Dpjovs & $\mathrm{LM}[\operatorname{Var}(\mathrm{u})=0, \rho=0)=122,22$ & $\mathrm{P}(0,00)$ \\
\hline
\end{tabular}

Para confirmar estes resultados foram efetuados mais dois outros testes. $\mathrm{O}$ primeiro, proposto por Greene (2000), testa se os erros possuem comportamento homocedástico. Este teste considera a especificação de um modelo do tipo efeitos fixos e testa a hipótese de que as variâncias são iguais para os grupos. O teste rejeitou em todas 
as especificações a hipótese nula de homocedasticidade. $\mathrm{O}$ segundo, proposto por Wooldridge (2002), verifica se os erros do modelo de efeitos fixos, anteriormente especificados, são isentos de autocorrelação de primeira ordem. O resultado do teste para os modelos especificados também rejeitou a hipótese nula de não-autocorrelação de primeira ordem. Como ambos os testes confirmaram os resultados apresentados na Tabela 2, opta-se por não reportá-los.

Os resultados dos testes indicam como mais adequado o modelo econométrico de efeitos fixos, mas este deve levar em consideração a autocorrelação e a heterocedasticidade na sua estimação. O modelo proposto por Kiefer (1980) atende estes perfis, pois estima um modelo em que os efeitos fixos (heterogeneidade dos Estados) são considerados juntamente com a autocorrelação. Este modelo consiste em aplicar o método MQGF - Mínimos Quadrados Generalizados Factíveis (FGLS-Feasible Generelized Least Square) sobre a seguinte equação:

$$
y_{i t}-\overline{y_{i}}=\left(x_{i t}-\bar{x}_{i}\right) \beta+\left(u_{i t}-\overline{u_{i}}\right)
$$

Note que $\bar{y}_{i}=T^{-1} \sum_{i=1}^{T} y_{i t}, \bar{x}_{i}=T^{-1} \sum_{i=1}^{T} x_{i t}$ e $\bar{u}_{i}=T^{-1} \sum_{i=1}^{T} u_{i t}$. Sob esta diferenciação, o termo $\xi_{i}$, que se refere aos aspectos individuais ou efeitos fixos de cada Estado, tende a ser minimizado. Este método consiste, portanto, em estimar uma matriz a priori de variâncias e covariâncias que é utilizada como uma matriz de pesos na obtenção dos parâmetros $\beta$ 's. ${ }^{5}$ Os resultados das estimações, usando este método, encontram-se na Tabela 3 .

As colunas (2) a (4) da Tabela 3 apresentam as variáveis de forma direta, enquanto as colunas (5) a (7) de forma proporcional. Para facilitar a interpretação, faremos uma análise dos efeitos das variáveis independentes sobre as dependentes entre os conjuntos de regressões. O nível de desenvolvimento tecnológico e de capital por pessoa (Pibh) é significante em todas as regressões. Portanto, o grau de desenvolvimento tecnológico da economia tem contribuído para reduzir o nível de analfabetismo e incentivar os jovens a frequentar o primeiro e o segundo graus, colunas (2) a (4). A confirmação deste resultado também aparece nas colunas de (5) a (7). Assim, os resultados demonstram que as proporçôes entre os analfabetos e estudantes de primeiro grau reduzem com o grau de desenvolvimento tecnológico, a proporção de jovens com educação superior a oito anos em relação aos analfabetos aumenta e, principalmente, aumenta a proporção

5 O estimador dos parâmetros consiste no seguinte: $\hat{\beta}=\left(\sum_{i=1}^{N} x_{i}^{\prime} \Theta^{-1} x_{i}\right)\left(\sum_{i=1}^{N} x_{i}^{\prime} \Theta^{-1} y_{i}\right)$. A matriz de pesos $\Theta$ é obtida através do estimador $\hat{\Theta}=N^{-1} \sum_{i=1}^{N} \hat{v}_{i} \hat{v}_{i}^{\prime} \operatorname{com} \hat{v}_{i}$ igual ao resíduo dos MQ - Mínimos Quadrados da regressão de primeiro estágio dos dados em conjunto, seccional e temporal. 
de jovens com maior escolaridade. Este último resultado é importante, pois revela que o nível de desenvolvimento está associado a uma busca por maior escolaridade pelos jovens, conforme previsto pelo modelo. Ou seja, as condiçóes atuais de mercado prevalecentes nas economias dos Estados, as instituições privadas, são importantes na decisão de acumular conhecimento dos jovens.

Os coeficientes da eficiência governamental (Efgov) nas regressões medem as variaçôes nos gastos de administrar como proporção dos demais gastos governamentais. Estas variações nos gastos administrativos ou eficiência governamental, como foram denominadas, não influenciam a taxa de analfabetismno ou o percentual de jovens frequentando o primeiro grau, colunas (2) e (3) da Tabela 3. No entanto, na coluna (4) da mesma tabela, esta parece ter efeito contrário no estímulo para os jovens obterem escolaridade acima de oito anos, pois o coeficiente $-0,65$ é significativo e forte indicador do efeito negativo. As colunas (5) a (7) implicam que, quando consideradas em conjunto as variáveis e na forma de proporção, a eficiência governamental influencia negativamente a acumulação de capital humano dos jovens. Provavelmente, isto se deve ao fato de que, maior gasto administrativo proporcional aos demais gastos governamentais significa menor investimento em educação, em especial de segundo grau e/ou superior, e/ou ainda, estes gastos geram demanda por trabalho de jovens em detrimento da acumulação de conhecimento. Além disso, este pode ser consequência de os gastos administrativos gerarem demanda por trabalhador não-qualificado e, portanto, agir como um fator contrário à acumulação de conhecimento, conforme preconiza Barros et alii (2001).

Além do mais, a análise da Tabela 3 revela que os investimentos em educação (Educinvpc) têm efeito positivo somente na redução do nível de analfabetismo, coluna (2). Assim, estes investimentos não estão exercendo o seu papel de incentivador adicional na obtenção da educação de primeiro grau, coluna (3), e se mostram negativos na obtenção de educação em nível de segundo grau, conforme coluna (4). Este resultado é confirmado quando estimamos as proporções. Somente a proporção de analfabetos e jovens com mais de oito anos de escolaridade (Dpanalfa) apresentase como significante e negativo, ou seja, os investimentos em educação reduzem a proporção entre analfabetos e alunos que frequentam o primeiro grau, coluna (5). $\mathrm{Na}$ última coluna, o efeito sobre a proporção de jovens com oito anos de escolaridade sobre os que frequentam o primeiro grau é claramente negativa. Este resultado demonstra que as políticas de investimento em educação na qualificação futura dos jovens devem ser revistas e rediscutidas amplamente, pois na sua forma atual não estão corroborando para aumentar a acumulação de conhecimento dos jovens. 


\section{TABELA 3 - RESULTADO DO MÉTODO MQGF}

\begin{tabular}{|c|c|c|c|c|c|c|}
\hline $\begin{array}{l}\text { Variáveis } \\
\text { (1) }\end{array}$ & $\begin{array}{l}\text { Analfa } \\
(2)\end{array}$ & $\begin{array}{l}\text { Pjovpg } \\
(3)\end{array}$ & $\begin{array}{c}\text { Pjovsec } \\
(4)\end{array}$ & $\begin{array}{c}\text { Dpanalfa } \\
(5)\end{array}$ & $\begin{array}{c}\text { Dpjovsec } \\
(6)\end{array}$ & $\begin{array}{c}\text { Dpjovs } \\
(7)\end{array}$ \\
\hline Pibh & $\begin{array}{l}-16,05 \\
(1,53)^{\star}\end{array}$ & $\begin{array}{c}2,10 \\
(0,92)^{\star *}\end{array}$ & $\begin{array}{c}7,88 \\
(1,58)^{\star}\end{array}$ & $\begin{array}{c}-0,20 \\
(0,02)^{*}\end{array}$ & $\begin{array}{c}1,41 \\
(0,22)^{*}\end{array}$ & $\begin{array}{c}0,09 \\
(0,02)^{*}\end{array}$ \\
\hline Efgov & $\begin{array}{c}-0,31 \\
(0,33)\end{array}$ & $\begin{array}{c}0,12 \\
(0,15)\end{array}$ & $\begin{array}{c}-0,65 \\
(0,25)^{\star}\end{array}$ & $\begin{array}{c}0,005 \\
(0,004)\end{array}$ & $\begin{array}{c}0,20 \\
(0,05)^{*}\end{array}$ & $\begin{array}{c}-0,007 \\
(0,002)^{* *}\end{array}$ \\
\hline Educinvpc & $\begin{array}{c}-7,49 \\
(3,46)^{\star *}\end{array}$ & $\begin{array}{c}2,69 \\
(1,96) \\
\end{array}$ & $\begin{array}{c}-7,81 \\
(3,06)^{*}\end{array}$ & $\begin{array}{l}-0,011 \\
(0,04)^{*}\end{array}$ & $\begin{array}{c}0,87 \\
(0,66)\end{array}$ & $\begin{array}{c}-0.096 \\
(0,034)^{\star}\end{array}$ \\
\hline Gini & $\begin{array}{c}33,19 \\
(7,07)^{\star}\end{array}$ & $\begin{array}{l}-15,68 \\
(4,23)^{*}\end{array}$ & $\begin{array}{l}-44,42 \\
(6,42)^{*}\end{array}$ & $\begin{array}{c}0,35 \\
(0,09)^{*}\end{array}$ & $\begin{array}{c}-2,94 \\
(0,98)^{*}\end{array}$ & $\begin{array}{l}-0,490 \\
(0,07)^{*}\end{array}$ \\
\hline Txresc & $\begin{array}{c}3,77 \\
(0,73)^{\star}\end{array}$ & $\begin{array}{l}-0,30 \\
(0,39)\end{array}$ & $\begin{array}{c}-2,83 \\
(0,73)^{*}\end{array}$ & $\begin{array}{c}0,05 \\
(0,009)^{\star}\end{array}$ & $\begin{array}{l}-0,95 \\
(0,16)\end{array}$ & $\begin{array}{c}-0,032 \\
(0,008)^{*}\end{array}$ \\
\hline Txresc2 & $\begin{array}{c}-4,94 \\
(0,87)^{\star}\end{array}$ & $\begin{array}{c}1,60 \\
(0,48)^{\star}\end{array}$ & $\begin{array}{c}5,21 \\
(0,78)^{*}\end{array}$ & $\begin{array}{c}-0,066 \\
(0,011)^{\star}\end{array}$ & $\begin{array}{c}0,48 \\
(0,21)^{\star *}\end{array}$ & $\begin{array}{c}0,051 \\
(0,008)^{*}\end{array}$ \\
\hline Educ25c11 & $\begin{array}{c}-0,81 \\
(0,20)^{\star}\end{array}$ & $\begin{array}{c}0,41 \\
(0,10)^{*}\end{array}$ & $\begin{array}{c}1,16 \\
(0,12)^{*}\end{array}$ & $\begin{array}{c}-0,012 \\
(0,002)^{\star}\end{array}$ & $\begin{array}{c}0,20 \\
(0,03)^{*}\end{array}$ & $\begin{array}{c}0,0109 \\
(0,001)^{*}\end{array}$ \\
\hline Constante & $\begin{array}{c}0,72 \\
(0,47)\end{array}$ & $\begin{array}{c}-0,37 \\
(0,22)^{\star \star \star}\end{array}$ & $\begin{array}{c}-2,49 \\
(0,30)^{*}\end{array}$ & $\begin{array}{c}0,013 \\
(0,005)^{\star \star}\end{array}$ & $\begin{array}{l}-0,22 \\
(0,05)\end{array}$ & $\begin{array}{c}-0,024 \\
(0,003)^{*}\end{array}$ \\
\hline$N^{*} T$ & 270 & 270 & 270 & 270 & 270 & 270 \\
\hline Chi2(7) & 428,43 & 136,28 & $1.894,95$ & 435,22 & 302,76 & $1.481,13$ \\
\hline
\end{tabular}

Obs.: * é o nível de significância inferior a $1 \%,{ }^{* *}$ inferior a $5 \% \mathrm{e}^{* * *}$ inferior a $10 \%$; Variáveis entre parênteses referem-se aos desvios padrão; $N$ é o número de painéis (27) e $T$, o período (10).

As distorções na distribuição de renda, representadas pelo índice de Gini, apresentam-se significativas em todas as regressões da Tabela 3, e sua análise não deixa dúvidas de que este representa um dos fatores preponderantemente inibidores à acumulação de conhecimento ou qualificação dos jovens. De acordo com a análise aqui apresentada, a razão para este resultado é que o índice de Gini capta as distorções do mercado de trabalho, ou seja, este representa a divisão do resultado gerado na produção. Além disso, tal situação pode ser ainda devida ao efeito barreira mencionado na introdução, onde um grupo é beneficiado com a transferência do Estado na forma de capital humano, o que gera a posteriori distorções no recebimento dos rendimentos proporcionais ao nível de educação. Como não existe equidade no acesso à educação, tem-se um processo desestimulador para a acumulação de capital humano futuro. Portanto, este resultado justifica o uso de políticas sociais de redistribuição de renda condicionadas ao fator de acumulação futura de educação dos jovens.

Em síntese, a combinação dos resultados dos impactos negativos da ineficiência governamental, mau desempenho dos investimentos em educação e do índice de distribuição de renda Gini, produz um resultado geral preocupante sobre o papel das instituições governamentais representadas pelas políticas públicas realizadas nos 
Estados. Portanto, políticas pró-ativas que melhorem o impacto destas variáveis de forma positiva para a acumulação de capital humano devem ser encontradas e aplicadas urgentemente.

Ainda com relação à Tabela 3 , as variáveis taxa média de retorno do capital humano (Txresc), seu valor ao quadrado (Txresc2) e escolaridade média acima de 11 anos das pessoas com mais de 25 anos (Educ25cl1) devem ser analisadas em conjunto. Estas variáveis atuam como se fossem desvios em relação à constante da regressão, que representa a média de acumulação de capital humano de longo prazo. Mais especificamente, estas variáveis capturam os efeitos fixos ou as características individuais de cada Estado - heterogeneidade das instituições privadas ou mercados para capital humano dos Estados. A variável Txresc captura as diferenças de cada mercado na alocação de capital humano através da sua taxa de retorno efetiva. No entanto, esta variável possui sinal positivo em relação ao analfabetismo e negativo em relação às demais. Isto demonstra que a taxa de retorno média atual não é um dos incentivadores da educação. Porém, ao se considerar seu valor ao quadrado, uma melhoria na taxa de retorno deste mesmo capital humano, por exemplo, pode ser resultado de uma política de redução de distorções no mercado de alocação de capital humano, trazendo benefícios positivos para acumulação de capital humano.6 Assim, o estoque de capital humano altamente qualificado, ou seja, de pessoas com mais de 25 anos com escolaridade superior a 11 anos $(E d u c 25 c 11)$, tem um papel importante na determinação da diferença entre os Estados e suas políticas de incentivo a qualificação dos jovens. Este efeito positivo é retroalimentador; os futuros jovens qualificados, quando adultos, irão auxiliar a aumentar a qualificação dos jovens do futuro. Dessa forma, o impacto destas variáveis é mensurado por meio da seguinte equação:

$$
\xi_{i}=\text { const }+\beta_{1}(\text { Txresc })+\beta_{2}(\operatorname{Tresc} 2)+\beta_{3}(\text { Educ } 25 \text { c11 })
$$

Note que const refere-se à constante de cada regressão, e os $\beta$ 's, aos parâmetros estimados nas regressões da Tabela 3. A equação (8) deve ser interpretada como uma taxa de crescimento média anual que é independente das políticas dos Estados e do seu grau de desenvolvimento. Por simplificação, os cálculos foram efetuados somente para as colunas (5) a (7) da Tabela 3 , pois estas se referem às variáveis de maior interesse, que são as taxas de crescimento das proporcionalidades dos indivíduos na busca por qualificação ou acumulação de capital humano. O primeiro caso, coluna (5) da Tabela 3, equivale à taxa de redução da percentagem de analfabetos sobre jovens no primeiro grau. O segundo caso, coluna (6) da mesma tabela, à taxa de aumento

6 As distorções de mercado podem ser entendidas como a legislação trabalhista. No entanto, pesquisas devem ser efetuadas para melhor se compreender quais são efetivamente as distorções existentes que desestimulam a acumulação de capital humano, uma vez que este não é o objetivo deste trabalho. 
da proporcionalidade de jovens no secundário sobre os analfabetos. O terceiro caso refere-se à taxa de crescimento dos jovens secundários sobre os de primeiro grau. Portanto, na Tabela 4, as taxas computadas derivam-se da influência das instituições que representam o mercado de capital humano.

Os resultados da equação (8) encontram-se na Tabela 4. Dessa forma, observa-se que São Paulo é o Estado que possui a maior taxa endógena de redução da proporção de analfabetos/jovens no primeiro grau, $-3,36 \%$, independente de suas políticas públicas e grau de desenvolvimento. Além disso, é o Estado que tem a melhor taxa natural de qualificação dos jovens frequentando o segundo grau como proporção dos analfabetos, $21,17 \%$. Com relação ao acréscimo de jovens no segundo grau em proporção aos de primeiro grau, a taxa endógena de crescimento é de $2,65 \%$. No outro extremo, Roraima apresenta-se com as seguintes taxas, que independem de suas políticas públicas: $-1,24 \%, 6,54 \%$ e $0,98 \%$, respectivamente, para a redução da proporcionalidade de analfabetos/estudantes de primeiro grau e de estudantes de segundo grau/analfabetos e primeiro grau, portanto, bem abaixo da média nacional.

O resultado principal da Tabela 4 é a heterogeneidade do mercado de alocação de capital humano dos Estados brasileiros. Esta heterogeneidade representa as diferenças existentes entre os Estados brasileiros na acumulação de conhecimento, que devem ser eliminadas com as políticas de investimento em educação e eficiência governamental, o que denominamos de instituições públicas. No entanto, conclui-se que as instituições públicas não estão desempenhando seu papel complementar de estimular a acumulação de capital humano dos jovens. Portanto, com a persistência do predomínio no longo prazo do efeito de mercado, instituição privada, em conjunto com a influência do estoque de capital humano qualificado podemos ter como resultado a manutenção da atual distribuição do conhecimento nos Estados. No entanto, como as taxas de crescimento da Tabela (4) diferem entre os Estados, haverá provavelmente um aumento na disparidade da diferença de estoque de capital humano entre os Estados no tempo e, portanto, aumentando ainda mais as suas diferenças no longo prazo. Para confirmar este resultado, aplicamos a seguir um método mais robusto na correção da heterogeneidade dos dados. 
TABELA 4 - INFLUENCIA DO MERCADO DE CAPITAL HUMANO E DO ESTOQUE DE CAPITAL HUMANO DOS ESTADOS BRASILEIROS

\begin{tabular}{|c|c|c|c|c|}
\hline Ordem & Estado & Dpanalfa & Dpjovsec & Dpjovs \\
\hline 1 & Acre & $-1,31 \%$ & $7,06 \%$ & $1,03 \%$ \\
\hline 2 & Alagoas & $-1,74 \%$ & $9,76 \%$ & $1,37 \%$ \\
\hline 3 & Amapá & $-1,40 \%$ & $7,73 \%$ & $1,11 \%$ \\
\hline 4 & Amazonas & $-1,87 \%$ & $10,54 \%$ & $1,47 \%$ \\
\hline 5 & Bahia & $-2,48 \%$ & $14,46 \%$ & $1,95 \%$ \\
\hline 6 & Ceará & $-2,20 \%$ & $12,74 \%$ & $1,73 \%$ \\
\hline 7 & Distrito Federal & $-2,07 \%$ & $13,52 \%$ & $1,65 \%$ \\
\hline 8 & Espírito Santo & $-1,95 \%$ & $11,32 \%$ & $1,54 \%$ \\
\hline 9 & Goiás & $-2,16 \%$ & $12,67 \%$ & $1,71 \%$ \\
\hline 10 & Maranhão & $-2,02 \%$ & $11,3 \%$ & $1,59 \%$ \\
\hline 11 & Mato Grosso & $-1,89 \%$ & $10,88 \%$ & $1,49 \%$ \\
\hline 12 & Mato Grosso do Sul & $-1,77 \%$ & $10,37 \%$ & $1,40 \%$ \\
\hline 13 & Minas Gerais & $-2,76 \%$ & $16,64 \%$ & $2,17 \%$ \\
\hline 14 & Pará & $-2,15 \%$ & $12,36 \%$ & $1,69 \%$ \\
\hline 15 & Paraíba & $-1,82 \%$ & $10,53 \%$ & $1,44 \%$ \\
\hline 16 & Paraná & $-2,46 \%$ & $14,84 \%$ & $1,94 \%$ \\
\hline 17 & Pernambuco & $-2,22 \%$ & $13,17 \%$ & $1,76 \%$ \\
\hline 18 & Piauí & $-1,75 \%$ & $9,69 \%$ & $1,38 \%$ \\
\hline 19 & Rio de Janeiro & $-2,63 \%$ & $16,55 \%$ & $2,08 \%$ \\
\hline 20 & Rio Grande do Norte & $-1,82 \%$ & $10,41 \%$ & $1,44 \%$ \\
\hline 21 & Rio Grande do Sul & $-2,46 \%$ & $14,98 \%$ & $1,94 \%$ \\
\hline 22 & Rondônia & $-1,57 \%$ & $8,60 \%$ & $1,23 \%$ \\
\hline 23 & Roraima & $-1,24 \%$ & $6,54 \%$ & $0,98 \%$ \\
\hline 24 & Santa Catarina & $-2,20 \%$ & $13,02 \%$ & $1,74 \%$ \\
\hline 25 & São Paulo & $-3,36 \%$ & $21,17 \%$ & $2,65 \%$ \\
\hline 26 & Sergipe & $-1,65 \%$ & $9,29 \%$ & $1,30 \%$ \\
\hline \multirow[t]{2}{*}{27} & Tocantins & $-1,57 \%$ & $8,44 \%$ & $1,24 \%$ \\
\hline & Média & $-2,02 \%$ & $11,80 \%$ & $1,59 \%$ \\
\hline
\end{tabular}

Fonte: Calculado usando a equação (8) e base de dados desta pesquisa.

\section{ANÁLISES DE ROBUSTEZ DOS RESULTADOS}

Para confirmar os resultados da seção anterior, as estimativas são repetidas, mas outro método é utilizado, aplicado na estimação da equação (5). Conforme Judge $e t$ alii (1985), tal método, proposto por Prais e Winsten, é um estimador de Mínimos Quadrados Generalizados com uma matriz de pesos que usa o coeficiente de au- 
tocorrelação, $\rho$ estimado. ${ }^{7}$ A heterocedasticidade é corrigida utilizando estimativas robustas das variâncias.

Este método permite uma avaliação de forma criteriosa dos resultados anteriores, em função da redução do efeito da heterogeneidade nos resultados, presente nos dados, pois este método utiliza um sistema mais pormenorizado de correção da heterocedasticidade. Outro aspecto importante é que este método nos fornece o grau de explicação da variável dependente, ou seja, o coeficiente de determinação.

Assim, foram efetuadas as mesmas regressões do método anterior. Em geral, o método de Prais-Winsten produziu resultados similares. No entanto, aqui o objetivo é destacar as pequenas diferenças, com o intuito de confirmar a robustez dos resultados anteriores.

De acordo com a Tabela 5, o efeito do grau de desenvolvimento da economia (Pibh) manteve-se estatisticamente significante e equivalente ao resultado anterior. A eficiência governamental (Efgov) demonstra ser significante e influencia negativamente somente a razão entre jovens secundários e adultos analfabetos, coluna (6) da Tabela 5. Isto significa que a eficiência governamental, de acordo com a medida proposta, tem contribuído para que a acumulação de capital humano, dos jovens em nível de segundo grau proporcionalmente aos adultos analfabetos, não seja mais intensa do que as taxas obtidas na Tabela 4. Além disso, os investimentos em educação dos Estados (Educinvpc) parecem atingir mais diretamente a redução do analfabetismo adulto, colunas (2) e (5) da Tabela 5, do que nas estimativas anteriores. E, marginalmente, contribui para desestimular os jovens a continuar seus estudos em nível de segundo grau. O efeito da distribuição de renda, índice Gini, aparece como um fator de incentivo à não-acumulação de conhecimento e, portanto, a perpetuação da nãoqualificação. Estes resultados confirmam a necessidade de políticas mais adequadas e diretas de redistribuição de renda, que premiem a acumulação de conhecimento dos jovens.

As variáveis que representam as condições de mercado, Txresc, Txresc2, Educ25cll e const são estatisticamente idênticas aos resultados anteriores, o que valida os resultados da Tabela 4.

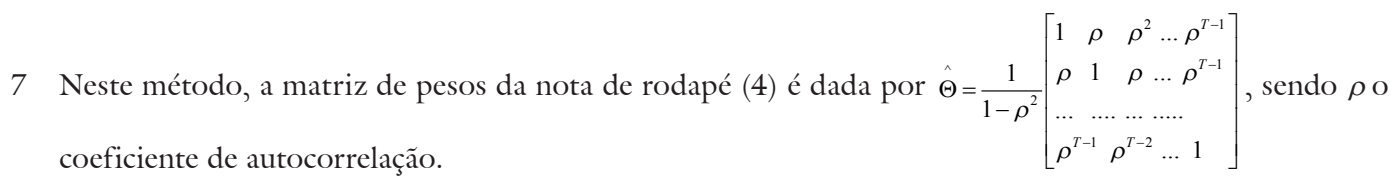


TABELA 5 - RESULTADO DO MÉTODO PWMQ

\begin{tabular}{|c|c|c|c|c|c|c|}
\hline $\begin{array}{l}\text { Variáveis } \\
\text { (1) }\end{array}$ & $\begin{array}{c}\text { Analfa } \\
(2)\end{array}$ & $\begin{array}{c}\text { Pjowpg } \\
(3)\end{array}$ & $\begin{array}{c}\text { Pjopsec } \\
(4)\end{array}$ & $\begin{array}{c}\text { Dpanalfa } \\
(5)\end{array}$ & $\begin{array}{c}\text { Dpjoosec } \\
(6)\end{array}$ & $\begin{array}{c}\text { Dpjows } \\
(7)\end{array}$ \\
\hline Pibh & $\begin{array}{l}-19,40 \\
(1,86)^{\star} \\
\end{array}$ & $\begin{array}{c}2,19 \\
(1,06)^{* *}\end{array}$ & $\begin{array}{c}8,90 \\
(2,22)^{*}\end{array}$ & $\begin{array}{c}-0.225 \\
(0.023)^{\star}\end{array}$ & $\begin{array}{c}1,422 \\
(0,303)^{\star}\end{array}$ & $\begin{array}{c}0,103 \\
(0,023)^{\star}\end{array}$ \\
\hline$E f g o v$ & $\begin{array}{l}-0,07 \\
(0,42) \\
\end{array}$ & $\begin{array}{l}0,022 \\
(0,23) \\
\end{array}$ & $\begin{array}{l}-0,49 \\
(0,35) \\
\end{array}$ & $\begin{array}{c}-0.0002 \\
(0.005) \\
\end{array}$ & $\begin{array}{c}-0,303 \\
(0,074)^{\star}\end{array}$ & $\begin{array}{l}-0,004 \\
(0,003) \\
\end{array}$ \\
\hline Educinvpc & $\begin{array}{l}-10,33 \\
(3,80)^{\star}\end{array}$ & $\begin{array}{c}2,75 \\
(2,30) \\
\end{array}$ & $\begin{array}{l}-8,16 \\
(5,12) \\
\end{array}$ & $\begin{array}{c}-0.141 \\
(0.044)^{\star}\end{array}$ & $\begin{array}{c}0,683 \\
(0,825)\end{array}$ & $\begin{array}{c}-0,101 \\
(0,056)^{\star \star}\end{array}$ \\
\hline Gini & $\begin{array}{l}40,81 \\
(8,08)^{\star}\end{array}$ & $\begin{array}{l}-16,76 \\
(5,21)^{*}\end{array}$ & $\begin{array}{l}-39,82 \\
(9,95)^{*}\end{array}$ & $\begin{array}{c}0.446 \\
(0.102)^{*}\end{array}$ & $\begin{array}{l}-7,565 \\
(1,77)^{\star}\end{array}$ & $\begin{array}{l}-0,415 \\
(0,03)^{*}\end{array}$ \\
\hline Txresc & $\begin{array}{c}3,74 \\
(0,86)^{\star}\end{array}$ & $\begin{array}{l}-0,30 \\
(0,45)\end{array}$ & $\begin{array}{c}-3,21 \\
(1,04)^{*}\end{array}$ & $\begin{array}{c}0.043 \\
(0.10)^{*}\end{array}$ & $\begin{array}{c}0,108 \\
(0,231)\end{array}$ & $\begin{array}{c}-0,035 \\
(0,011)^{\star}\end{array}$ \\
\hline Txresc2 & $\begin{array}{c}-5,85 \\
(1,00)^{*}\end{array}$ & $\begin{array}{c}1,50 \\
(0,63)^{\star}\end{array}$ & $\begin{array}{c}5,77 \\
(0,97)^{\star}\end{array}$ & $\begin{array}{c}-0.76 \\
(0.012)^{\star}\end{array}$ & $\begin{array}{c}0,919 \\
(0,273)^{\star}\end{array}$ & $\begin{array}{c}0,055 \\
(0,009)^{\star}\end{array}$ \\
\hline$E d u c 25 c 11$ & $\begin{array}{l}-0,35 \\
(0,25)\end{array}$ & $\begin{array}{c}0,41 \\
(0,11)^{\star}\end{array}$ & $\begin{array}{c}1,11 \\
(0,18)^{\star}\end{array}$ & $\begin{array}{c}-0.007 \\
(0.003)^{\star \star}\end{array}$ & $\begin{array}{c}0,207 \\
(0,037)^{\star}\end{array}$ & $\begin{array}{c}0,0106 \\
(0,002)^{*}\end{array}$ \\
\hline Constante & $\begin{array}{c}1,88 \\
(0,56)^{\star}\end{array}$ & $\begin{array}{l}-0,41 \\
(0,27) \\
\end{array}$ & $\begin{array}{c}-2,47 \\
(0,38)^{\star}\end{array}$ & $\begin{array}{c}0.024 \\
(0.006)^{\star}\end{array}$ & $\begin{array}{c}-0,261 \\
(0,083)^{\star} \\
\end{array}$ & $\begin{array}{c}-0,024 \\
(0,003)^{*}\end{array}$ \\
\hline$N^{*} T$ & 270 & 270 & 270 & 270 & 270 & 270 \\
\hline$R^{2}$ & 0,62 & 0,26 & 0,67 & 0,59 & 0,55 & 0,64 \\
\hline
\end{tabular}

Fonte: Cálculo dos autores.

Obs.: * é o nível de signficância inferior a $1 \%,{ }^{* *}$ inferior a $5 \% \mathrm{e}^{* * *}$ inferior a $10 \%$; variáveis entre parênteses referem-se aos desvios padrão; $N$ é o número de painéis (27) e $T$, o período (10).

\section{CONCLUSÃO}

Nesta conclusão queremos confrontar os nossos resultados obtidos nas estimativas econométricas com o papel das instituições apresentados na discussão da literatura e indicadas pelo modelo teórico. As diferenças encontradas nas taxas de acumulação de capital humano entre os Estados, devido às suas características de mercado e grau de desenvolvimento tecnológico, deveriam ser amenizadas pelas políticas educacionais e pela eficiência do governo. No entanto, as políticas educacionais parecem estar voltadas exclusivamente para o combate do analfabetismo, desconsiderando totalmente o maior retorno econômico e social de se investir nos jovens. Portanto, são contrárias ao recomendado pelo modelo teórico. Além do que, estas contribuem para a visão de uma persistência histórica de desigualdade entre classes sociais, ou seja, um grupo de pessoas somente é atendido no acesso à educação na condição de adulto. Este resultado parece ser confirmado pelo alto grau de significância do índice Gini. 
A eficiência da instituição governo, medida pelas variações nos seus custos, produz efeitos negativos na acumulação de conhecimento dos jovens. Esta medida de ineficiência, ainda que não ideal, deve ser vista como uma barreira que reduz o nível de acumulação de conhecimento dos jovens nos Estados brasileiros. Esta ineficiência também fomenta, na nossa visão, o aumento de rent seekers e reduz a proporção de empreendedores que formam o mercado para o capital humano, o que é refletido nas baixas taxas de retorno do investimento para este capital. Assim, as taxas de retornos efetivas médias do capital humano nos mercados, quando consideradas per se não são um fator estimulador da educação dos jovens no primeiro grau e apresentam-se como desincentivadoras da busca por uma escolaridade acima de oito anos.

No entanto, outra explicação para o resultado acima é que aumentos em gastos administrativos em relação aos demais gastos geram de alguma forma demanda por mão de obra com qualificação abaixo de oito anos de escolaridade junto ao mercado. Neste caso, há a clara necessidade de se adotar critérios que melhorem a eficiência dos gastos do governo.

Dessa forma, o fator de maior incentivo à acumulação de capital humano dos jovens é o estoque de capital humano qualificado, adultos com educação superior a 11 anos. Este estoque mais do que compensa os demais efeitos negativos. Este efeito produzido pelo nível educacional já acumulado domina os efeitos da razão das taxas de retorno e custo de obtenção da educação dos jovens.

No geral, os resultados parecem dar suporte à ideia de que as políticas públicas não estão contribuindo para o processo de acumulação de capital humano dos jovens; podem estar contribuindo para a manutenção do processo histórico de desigualdade ao educar adultos analfabetos; prejudicam a taxa de retorno de mercado do capital humano acumulado através de sua ineficiência nos gastos, que estimulam rent seekers e geram demanda por mão de obra não-qualificada. Contudo, a acumulação de conhecimento dos jovens ocorre somente por influência dos fatores como grau de desenvolvimento tecnológico e o estoque de capital humano existente, e, ainda, de forma muito desigual entre os Estados.

\section{REFERENCLIAS}

AHSAN, S. M. Growth, Governance and poverty: a cross-country analysis. UNU/ WIDER Conference, Helsink, 2001.

BALTAGI, B. H.; LI, Q. Testing AR(1) against MA(1) disturbances in an error component model. Journal of Econometrics, v. 68, p. 133-151, 1995. 
BARROS, R. P.; MENDONÇA, R. Investimento em educação e desenvolvimento econômico. In: A economia brasileira em perspectiva. Rio de Janeiro: IPEA, 1998.

; SANTOS, D. D.; QUINTAES, G. Determinantes do desempenho educacional no Brasil. Pesquisa e Planejamento Econômico, v. 31, n.1, p. 1-37, 2001.

BELTRÃO, K. I. Alfabetização por sexo e raça no Brasil: um modelo linear generalizado para explicar a evolução no periodo 1940-1990. IPEA, 2003. (Texto para Discussão, n. 1003).

COLEMAN, J. Social capital in the creation of human capital. American Journal of Sociology, v. 94, n. 1, p. S94-S120, 1998.

DIAS, J.; DIAS, M.H. Crescimento econômico, emprego e educação em uma economia globalizada. Maringá: EDUEM, 1999.

DIAS, J.; MCDERMOTT, J. Institutions, education, and development: the role of entrepreneurs. Journal of Development Economics, v. 80, n.1, p. 299-328, 2006.

GOMES, C. A.; SOBRINHO, J. A. Qualidade, eficiência e equidade na educação básica. Rio de Janeiro: IPEA, 1992.

GOODFRIEND, M.; MCDERMOTT, J. Early development. American Economic Review, v. 85, n. 2, p. 116-133, 1995.

GREENE, W. H. Econometric analysis. London: Prentice-Hall International, 2000.

Hall, R.; Jones, C. I. Why do some countries produce so much more output per worker than others? Quarterly Journal of Economics, v. 114, n. 1, p. 83-116, 1999.

HECKMAN, J. J. Policies to Foster Human Capital. Research in Economics, v. 54, n. 1, p. 3-56, 2000.

JUDGE, G. G.; GRIFFITHS, W. E.; HILL, R. C.; LUTKEPOHL, H.; LEE, T. C. The theory and practice of econometrics. New York, John Willey and Sons, 1985.

KIEFER, N. M. Estimating fixed effects models for time series of cross-section with arbitrary intertemporal covariance. Journal of Econometrics, v. 14, n. 1, p. 195-202, 1980.

LUCAS JR., R. J. On the mechanics of economic development. Journal of Monetary Economics, v. 22, n. 1, p. 3-42, 1988.

SOKOLOFF, K. L.; ENGERMAN, S. L. History lessons: institutions, factor endowments, and paths of development in the new world. The Journal of Economic Perspectives, v. 14, n. 3, p. 217-232, 2000.

TEMPLE, J. The new growth evidence. Journal of Economic Literature, v. 37, n. 1, p. 112-156, 1999.

WOOLDRIDGE, J. M. Econometric analysis of cross section and panel data. London: MIT Press, 2002. 